\title{
Research Paper \\ Frequency of Vectors of Methicillin-resistant Staphylococcus Aureus Among Emergency Staff of Vali-e-Asr Hospital in Arak City, 2018
}

\author{
Ramin Parvizrad $^{1} \odot$, *Sara Khalili Dermani ${ }^{2} \odot$, Azam Ahmadi²
}

1. Department of Emergency Medicine, School of Medicine, Arak University of Medical Sciences, Arak, Iran

2. Infectious Diseases Research Center, Arak University of Medical Sciences, Arak, Iran.

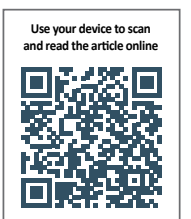

Citation: Parvizrad R, Khalili Dermani S, Ahmadi A. [Frequency of Vectors of Methicillin-resistant Staphylococcus Aureus Among Emergency Staff of Vali-e-Asr Hospital in Arak City, 2018 (Persian)]. Journal of Arak University of Medical Sciences (JAMS). 2020; 23(3):292-299. https://doi.org/10.32598/JAMS.23.3.5943.1

\section{https://doi.org/10.32598/JAMS.23.3.5943.1}

Key words:

Staphylococcus Emergency, Methicillin aureus, Colonization,

\section{A B S TRACT}

Article Info:

Received: 19 Jul 2019

Accepted: 04 May 2020

Available Online: 01 Aug 2020

\section{Extended Abstract}

\section{Introduction}

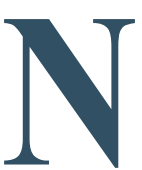

osocomial infections are a global problem and many factors are involved in its occurrence. The microorganisms that cause these infections are changing every year. Since the 1980s, gram-positive microorganisms, especially staphylococcus aureus, have emerged as a major cause of nosocomial infections [1].

Methicillin resistance is a type of chromosomal resistance caused by changes in PBP2a due to the mecA gene, which leads to a decrease in affinity for this type of PBP to beta-lactams. Organisms that have this type of resistance are called MRSA (Methicillin-Resistant Staphylococcus aureus) and are not affected by any of the beta-lactam antibiotics $[4,5]$.

\section{* Corresponding Author:}

Sara Khalili Dermani, MSc.

Address: Infectious Diseases Research Center, Arak University of Medical Sciences, Arak, Iran

Tel: +98 (918) 8645783

E-mail: sarakhalili89@yahoo.com 
Due to the importance of nosocomial infections, identifying and treating hospital staff who carry staphylococcus can reduce the incidence of methicillin-resistant staphylococci. This study was performed to determine the prevalence of carriers of methicillin-resistant staphylococcus aureus nasopharynx and bacterial contamination of cell phones' surfaces among emergency staff of Vali-e-Asr Hospital in Arak City.

\section{Materials and Methods}

This descriptive study was performed on the staff working in emergency ward of Vali-e-Asr Hospital in Arak City, 2018, after obtaining their informed consent. Exclusion criteria were the subjects' dissatisfaction and recent history of rhinoplasty. The subjects completed a questionnaire including demographic characteristics and history of antibiotic consumption in the past 3 weeks. After the subjects were present at work, samples of their nasal mucous discharge and surface of cell phones were collected. Samples were collected by soaking the cotton part of the swab with nasal mucous discharge and by rubbing a wet swab for 4 to 5 seconds on the entire surface of the mobile phone.

These samples were transferred to the TSB transport medium and sent to the laboratory within a maximum of 2 hours. These samples were cultured in plates containing mannitol salt agar (MSA) at $37^{\circ} \mathrm{C}$ for $48-72$ hours. The plates were examined after $18-24$ hours of incubation at $37^{\circ}$ $\mathrm{C}$ to isolate and identify gram-positive cocci. Staphylococcus strains were then isolated by standard microbiological methods (catalase, coagulase, mannitol fermentation and DNase). The susceptibility of the samples to the disc of cefoxitin and oxacillin was investigated and the presence of mecA gene in the mentioned strains was evaluated by polymerase chain reaction method.

\section{Results}

In this study, 70 employees of the emergency department of Vali-e-Asr Hospital in Arak City and their cell phones were tested. The results of culture of nasal mucous discharge and

a) $162 \mathrm{bp}$

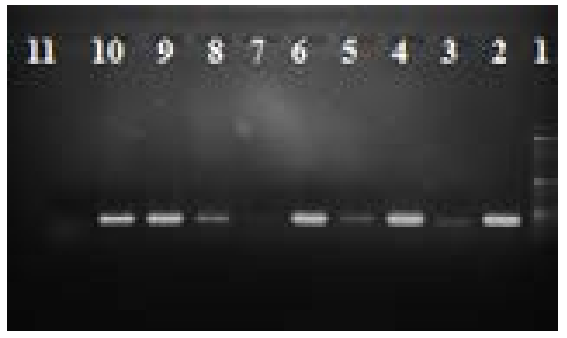

Figure 1. PCR results and Sa442 gene cell phone samples of these employees showed that 16 personnel and 3 cell phones were infected with staphylococcus aureus. The results of this study also showed that among the positive test samples, 11 cases of nasal mucous discharge and one case of mobile phone samples were MRSA and the other positive cases were MSSA. Molecular testing of all 19 positive samples also confirmed MRSA strains (Figure 1).

a. Evaluation of the mecA gene for confirmation of MRSA strains (line 1: bp 100 marker; line 2: positive control; lines 3-10: positive samples; line 11: negative control); b. Sa442 gene in isolated staphylococcus aureus on agarose gel (line 1: bp 100 marker; line 2: positive control; lines 3-9: positive samples; line 10: negative control, distilled water).

The results of this study showed that the occupational distribution of people infected with this bacterium included 11 nurses, 4 physicians and 1 service personnel.

\section{Discussion}

One of the basic principles in the control of nosocomial infections is the constant monitoring of the status of microorganisms in the hospital and the antibiotic resistance of these organisms [12]. In this study, out of 70 emergency personnel, $16(22.8 \%)$ were carriers of staphylococcus aureus, of which 11 samples $(15.7 \%$ of samples and $68.7 \%$ of carriers) were MRSA and 5 samples (7\% of samples and $31.3 \%$ of carriers) were MSSA. Also, staphylococcus aureus was isolated from three cell phones $(4.2 \%)$, one of which was MRSA.

Similar studies were performed on the frequency of carriers of methicillin-resistant staphylococcus aureus among hospital staff in Iran, which showed different results. For example, among the staff of Imam Khomeini Hospital in Tabriz City $37.4 \%$ [13], among the staff of clinical wards of Ali Ibn Abi Taleb Hospital in Rafsanjan City 77.3\% [14], among the staff of Namazi Hospital in Shiraz City 5.3\% [15], among the staff of Hazrat Masoumeh Hospital in Qom City 10.8\% [16], and in Shahrekord City 44\% [5] was reported.

b) $108 \mathrm{bp}$

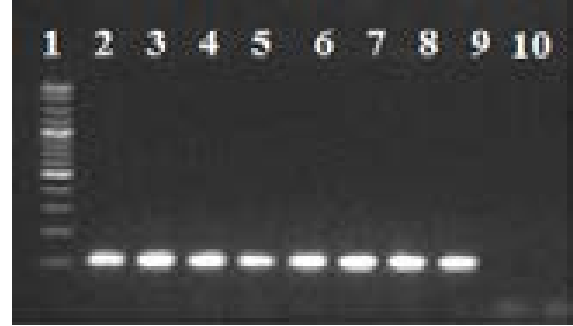


In a study conducted in 2016 among the staff of Qazvin Hospital, out of 198 samples, 32 people (16\%) were carriers of staphylococcus, the highest frequency $(20.3 \%)$ belonged to the intensive care unit staff, and the prevalence of MRSA in the total study population was 3\% [17]. In a study conducted in Tehran City, frequency of staphylococci carriers was $48.25 \%$ and MRSA was $10.82 \%$ [18]. In Sari City, the prevalence was $19.4 \%$ for staphylococci and $7.1 \%$ for MRSA [19]. Also in similar studies in other countries, the prevalence of methicillin-resistant Staphylococcus aureus among hospital staff in Turkey was $2.9 \%$ [20], in Toronto (Canada) 0\% [21], in Saudi Arabia 18\% [22], in France $6.2 \%$, in Ireland $7.5 \%$ and in New York (USA) $6.6 \%$.

In a study conducted in Tabriz City to investigate the microbial contamination of cell phones of Sina Hospital staff, $84.28 \%$ of cell phones were contaminated. The highest frequency of microorganisms was related to staphylococcus coagulase negative and bacillus species, and 3.57\% was related to staphylococcus aureus.

In general, the results of this study showed that the frequency of methicillin-resistant staphylococcus strains among the emergency staff of Vali-e-Asr Hospital in Arak City was significant and the highest infection was observed among the nurses in this ward.

\section{Ethical Considerations}

\section{Compliance with ethical guidelines}

This study was approved by the Research Ethics committee of Arak University of Medical Sciences .with code :

IR.ARAKMU.REC.1396.282

\section{Funding}

This research did not receive any grant from funding agencies in the public, commercial, or non-profit sectors.

\section{Authors' contributions}

All contributed in preparing this article.

\section{Conflicts of interest}

The authors declared no conflict of interest.

\section{Acknowledgements}

The authors would like to express their gratitude to the staff of the Infectious Diseases Research Center of Arak University of Medical Sciences who cooperated in carrying out this research. 


\title{
فراوانى ناقلين استافيلوكوكوس اورئوس مقاوم به متىسيلين در كاركنان اورثانس بيمارستان

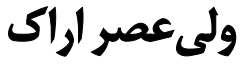

\author{
رامين برويزراد' •، •سارا خليلى درمنى ٪. اعظم احمدى'

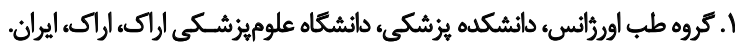

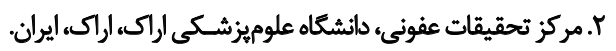

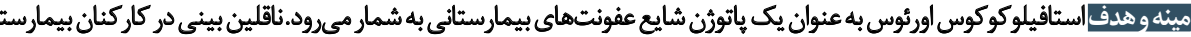

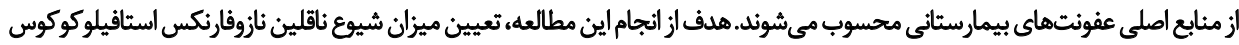

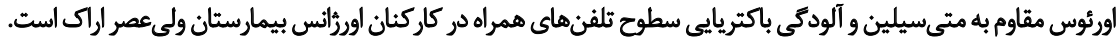

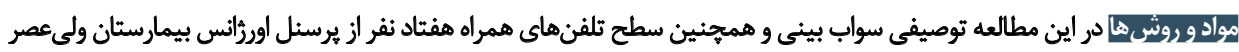

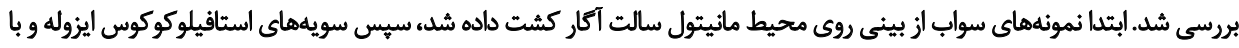

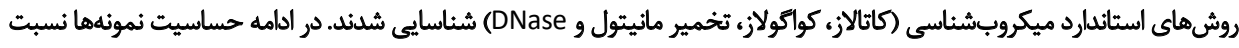

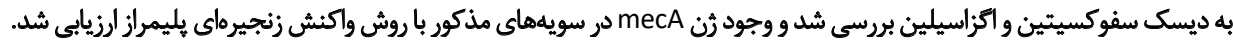

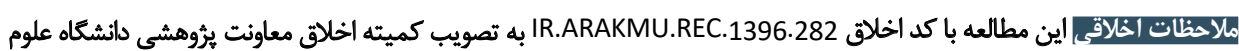
بز شكى اراى رسيد.

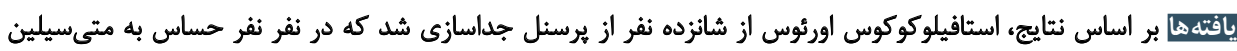

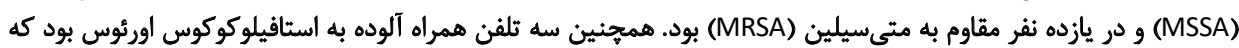

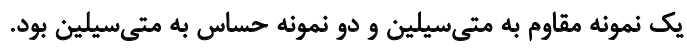

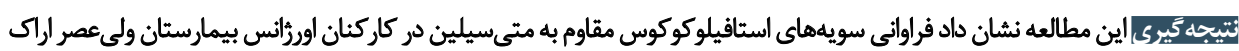

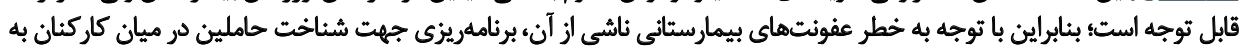

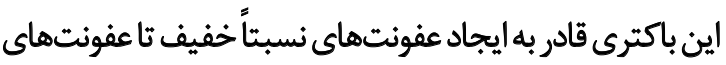

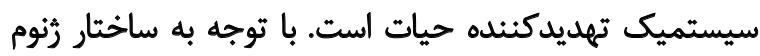

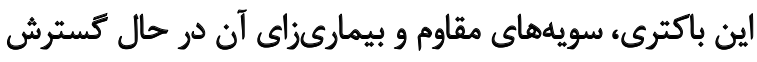

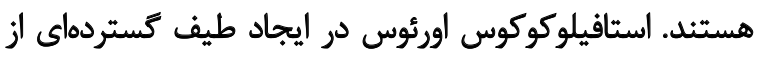

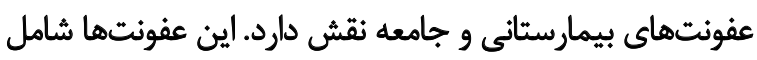

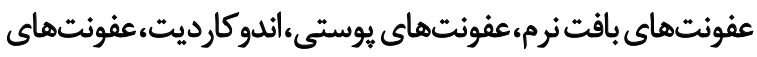

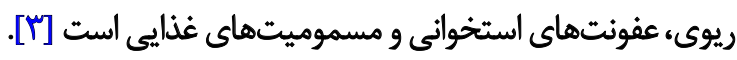
مقاومت به متىسيلين يك نوع مقاومت كروموزومال است

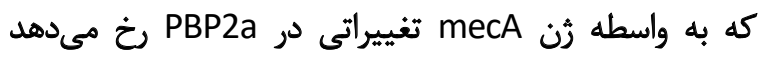

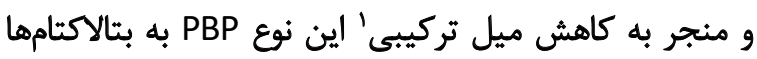

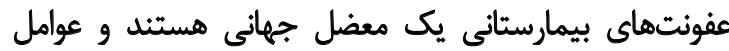

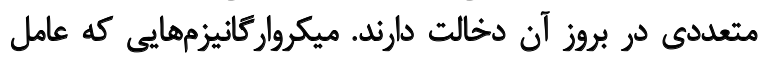

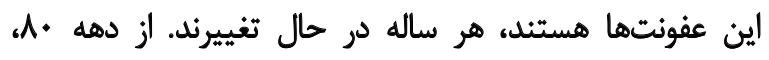

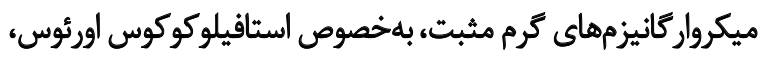

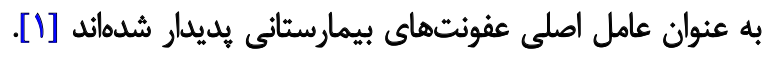

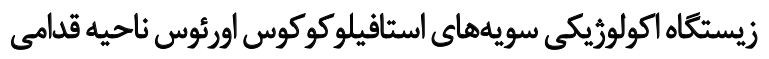

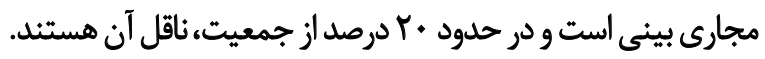

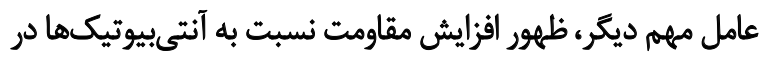

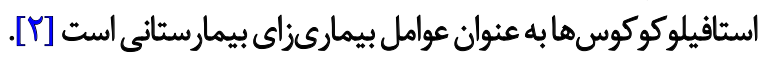

1. Affinity

ㄷ...

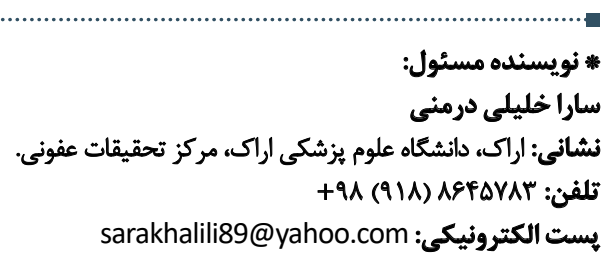


بيمارستان هستند. شيوع و انتشار حاملين MRSA برحسب نوع

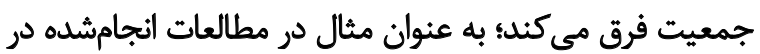

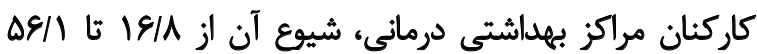

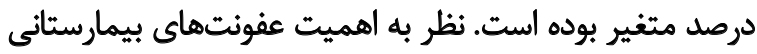

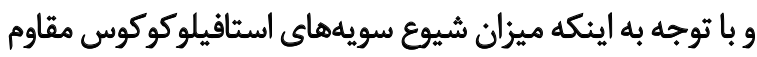

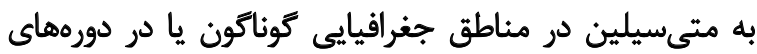

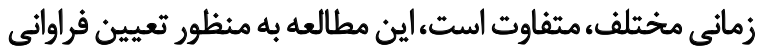

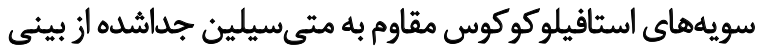

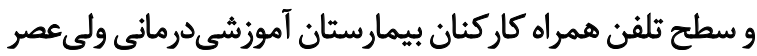

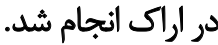

\section{مواد ورش روشها}

اين مطالعه توصيفى، در سال لوه"ا در بخش اورثانس

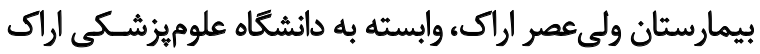

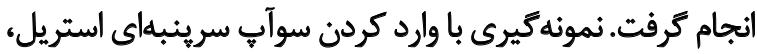

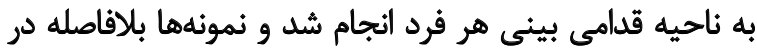

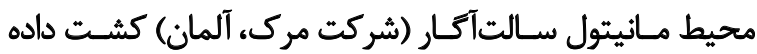

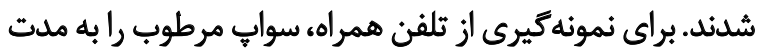

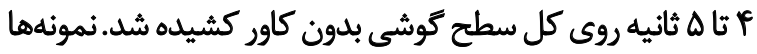

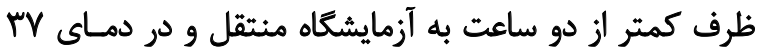

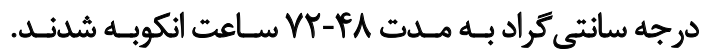

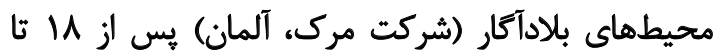

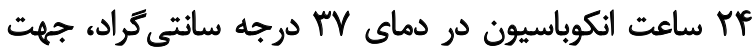

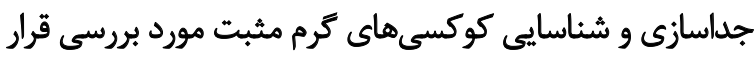

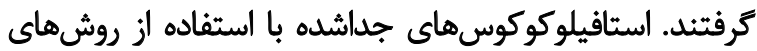

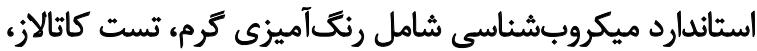

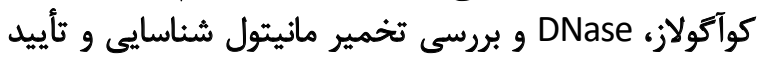

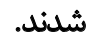

براى تعيين حساسيت نسبت به متىسيلين، تمامى نمونهها

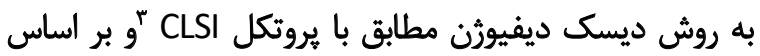

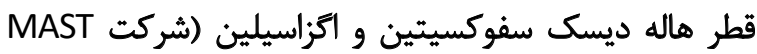
انعلستان) مورد بررسى قرار ترفتي

3. Clinical and laboratory standards institute

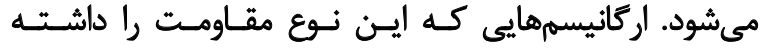

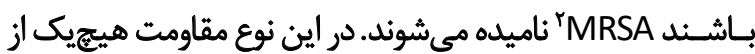

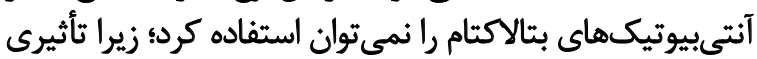

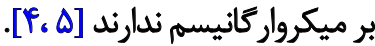

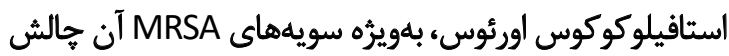

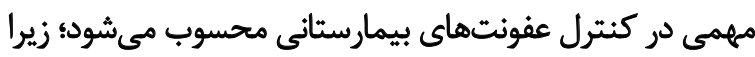

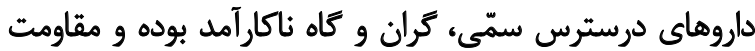

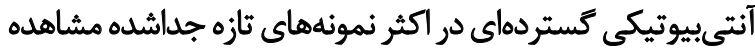

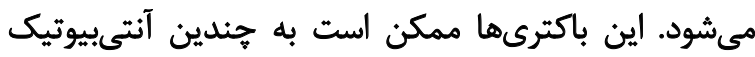

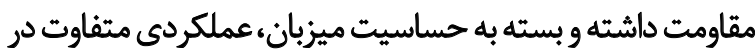

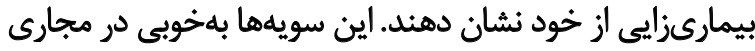

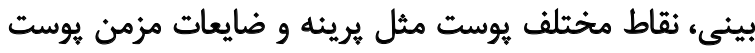

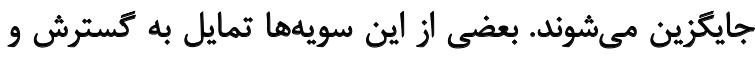

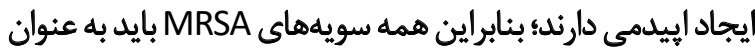

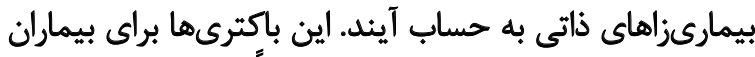

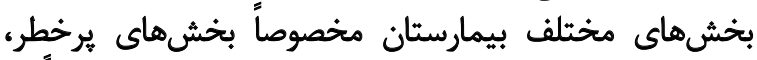

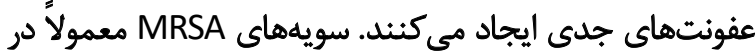

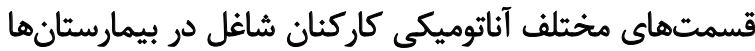

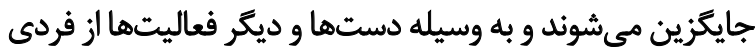

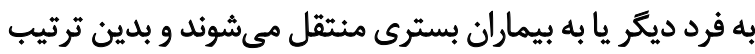

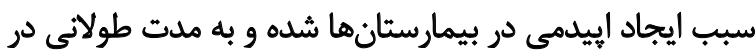

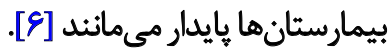

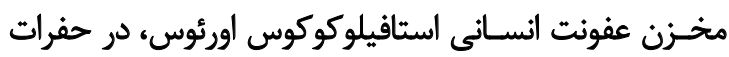

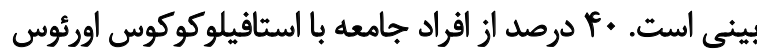

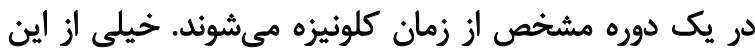

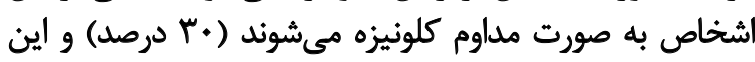

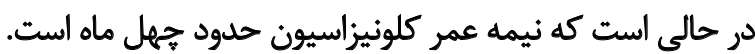

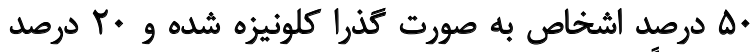

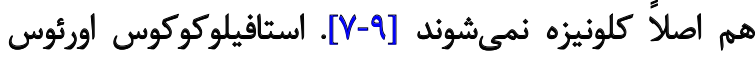

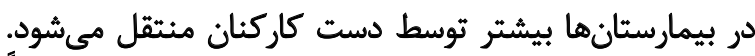

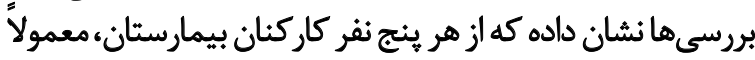

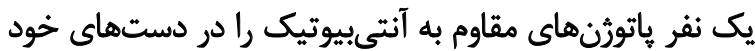

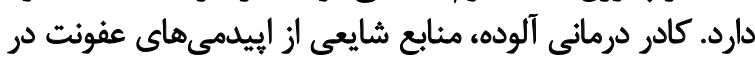

2. Methicillin-Resistant Staphylococcus aureus

جدول ا. مشخحصات يرايمرهاى استفاده شده جهت شناسايى ثُن هاى Sa442 وmecA

\begin{tabular}{|c|c|c|c|}
\hline ثام رُن & طول باند & توالى يرايمر & رفرئس \\
\hline Sa442 & $108 \mathrm{bp}$ & $\begin{array}{l}\text { AATCTTTGTCGGTACACGATATTCTTCACG } \\
\text { CGTAATGAGATTTCAGTAGATAATAACAAC }\end{array}$ & \multirow{2}{*}[1\cdot]{} \\
\hline mecA & $162 \mathrm{bp}$ & $\begin{array}{l}\text { TCCAGATTACAACTTCACCAGG } \\
\text { CCACTTCATATCTTGTAACG }\end{array}$ & \\
\hline
\end{tabular}


b) $108 \mathrm{bp}$

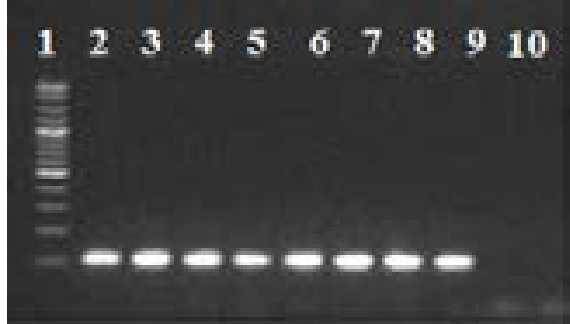

a) $162 \mathrm{bp}$

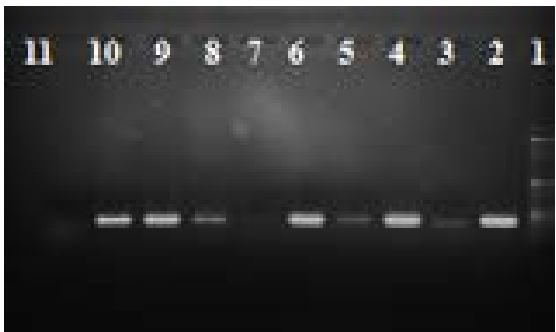

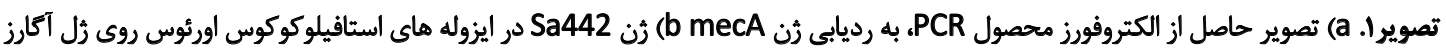

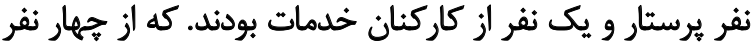

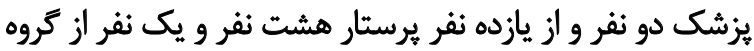

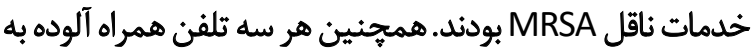

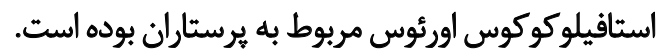

ث

يكى از اصول اوليه در كنترل عفونتهاى بيمارستائى نظارت

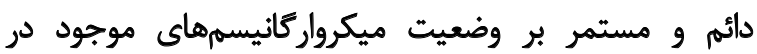

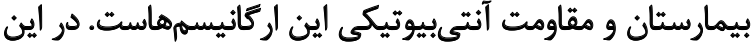

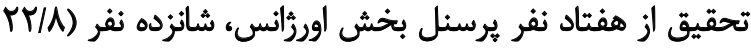

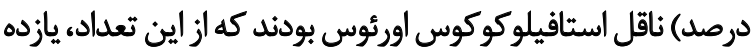

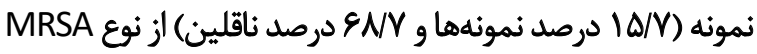

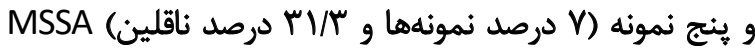

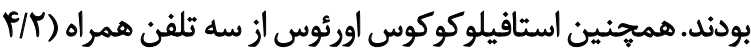

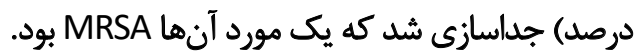

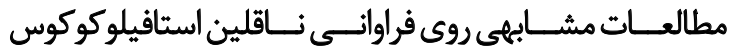
اورئوس مقاوم به متيىسيلين در يرســـنل بيمارستاني در ايران

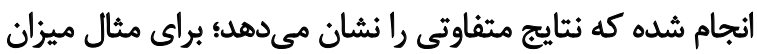

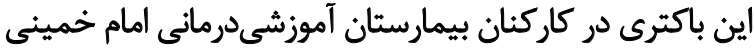

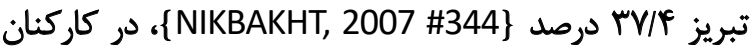
يخش هاي باليني بيمارستان على بن ابي طالب رفسنجان

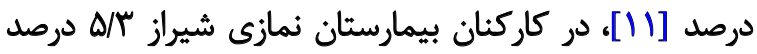

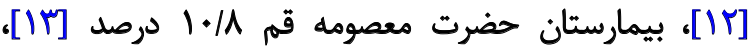

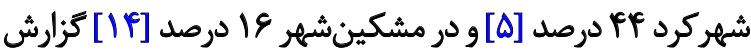
شلده است.

در كشورهاي ديعُ شيوع استافيلوكوكوس اورئوس مقاوم

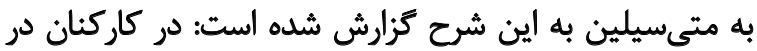

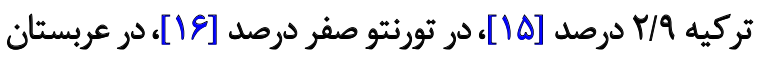

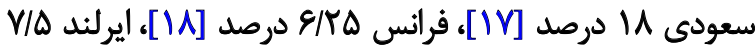

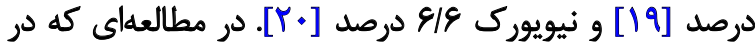

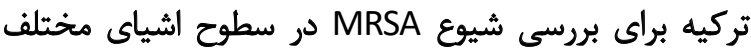
(تلفنهاي همراه، كوشى يزشكى، برى صئدلى، شير آب،كيبورد و
ماركي ارزيابي رُنوتيبى، جدايهها بر اساس ثرن Sa442، به عنوان

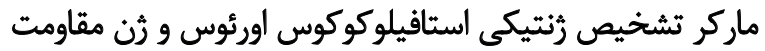

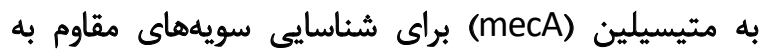

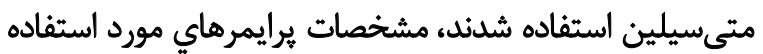

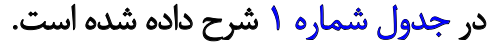

تكثير قطعه موردنظر با استفاده از دستعاه PCR با دماي

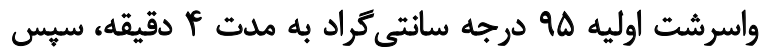

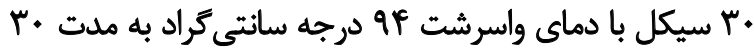

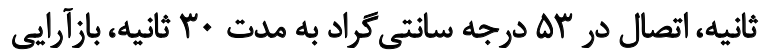

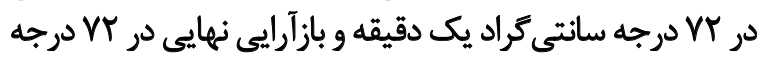

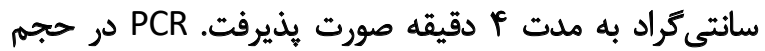

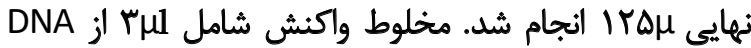

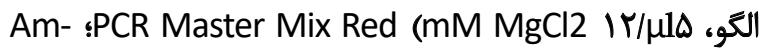

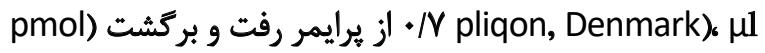

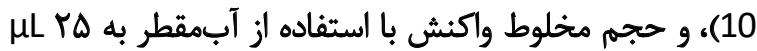

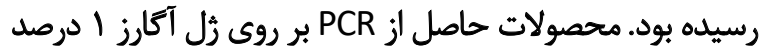

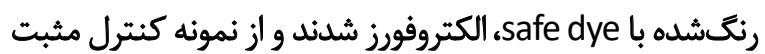
ATTC:49476 Staphylococcus Aureus

Ladil

در اين مطالعه هفتاد نفر از كاركنان بخش اورثانس در محدوده

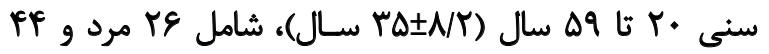

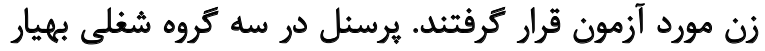

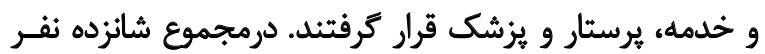

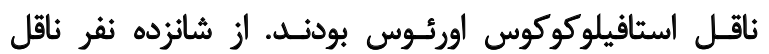
استافيلوكوكوس اورئوس، ينج نفر حساس به متى استيلين (MSSA)

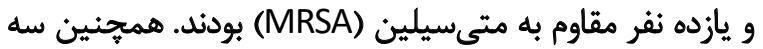

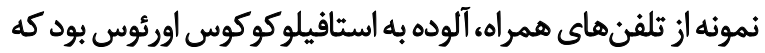

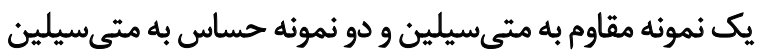

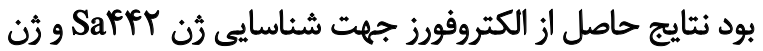

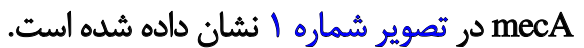

در بين افراد دارى كشت مثبت، جهار نفر يزشك، يازده 


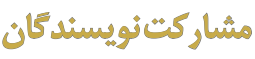

تمامــى نويســندكان در نــارش ايسـن مقالـهـ مشــاركت

داشـــتهاند.

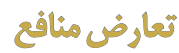

نويسندكان مقاله هيجَّونه تعارضى در منافع اعلام نكردند.

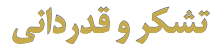

بدينوسيله نويسندكان اين مقاله از يرسنل محترم مركز

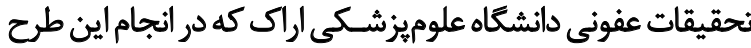
يرؤشى همكارى كردند، نهايت تشكر را دارند.

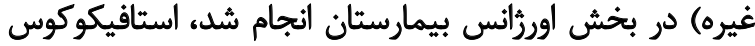

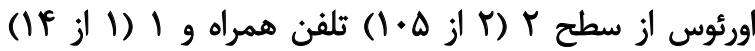

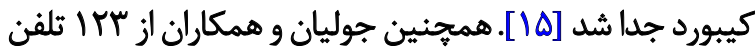

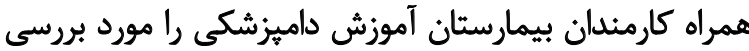

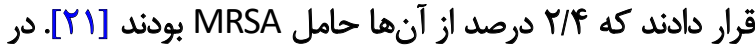

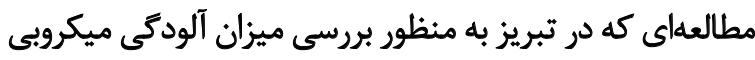

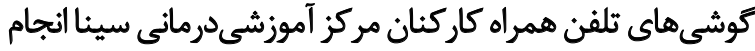

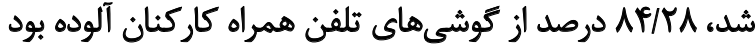

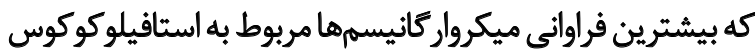

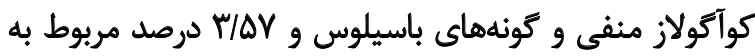

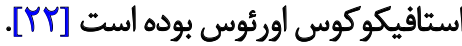

تفاوت جشمكير موجود بين حاملين استافيلوكوكوس اورئوس

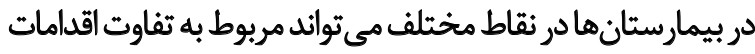

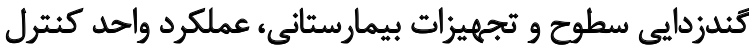

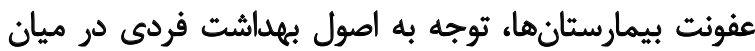

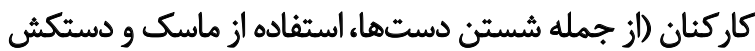

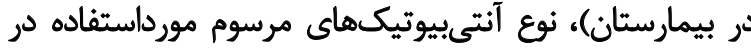

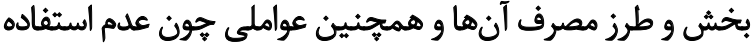

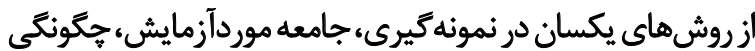
انجام آزمايش و نوع بيمارستان موردنظر باشد.

$$
\text { تتيجليكيرى }
$$

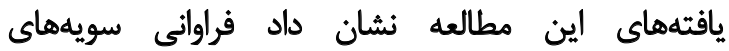

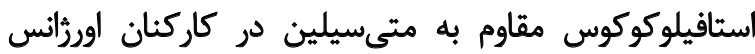

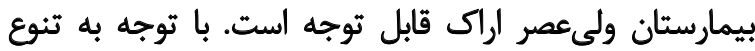

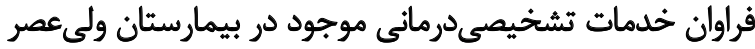

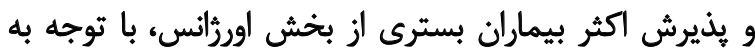

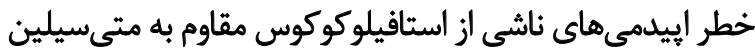

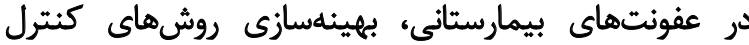

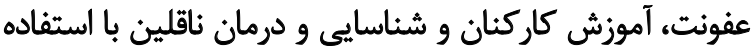

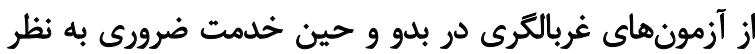

$$
\text { مىرسد. }
$$

ماحظات اخلاقي - الاتي

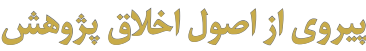

اين مطالعه با كد اخلاق

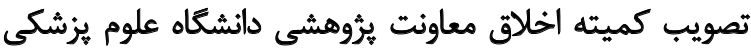

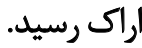

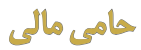

اين بثروهش حامى مالى نداشته است. 


\section{References}

[1] Kluytmans J, Van Belkum A, Verbrugh H. Nasal carriage of Staphylococcus aureus: Epidemiology, underlying mechanisms, and associated risks. Clin Microbiol Rev. 1997; 10(3):505-20. [DOI:10.1128/CMR.10.3.505] [PMID] [PMCID]

[2] Vandenbergh MF, Verbrugh HA. Carriage of Staphylococcus aureus: Epidemiology and clinical relevance. J Lab Clin Med. 1999; 133(6):525-34 [DOI:10.1016/S0022-2143(99)90181-6]

[3] Klevens RM, Morrison MA, Nadle J, Petit S, Gershman K, Ray S, et al. Invasive methicillin-resistant Staphylococcus aureus infections in the United States. JAMA. 2007; 298(15):1763-71. [DOI:10.1001/ jama.298.15.1763] [PMID]

[4] Braunwald E, Fauci AS, Kasper DL, Hauser SL, Longo DL, Jameson JL. Harrison's principle of internal medicine [CD-ROM]. New York: McGraw-Hill; 2001. https://www.amazon.com/Harrisons-Principles-Internal-Medicine-15th/dp/0070072728

[5] Kalhor H, Validi M, Nafisi MR. Evaluation of the frequency of MethicillinResistant Staphylococs isolated from nose of nursing personnel of Hajar Hospital of Shahrekord. Qom Univ Med Sci J. 2013; 7(1)42-9.

[6] Katayama $Y$, Zhang $H Z$, Hong $D$, Chambers HF. Jumping the barrier to $\beta$-lactam resistance in Staphylococcus aureus. J bacteriol. 2003; 185(18):5465-72. [DOI:10.1128/JB.185.18.54655472.2003] [PMID] [PMCID]

[7] Wertheim HF, Melles DC, Vos MC, Leeuwen WV, Belkum AV, Verbrugh HA, et al. The role of nasal carriage in Staphylococcus aureus infections. Lancet Infect Dis. 2005; 5(12):751-62. [DOI:10.1016/S14733099(05)70295-4]

[8] David MZ, Daum RS. Community-associated methicillin-resistant Staphylococcus aureus: Epidemiology and clinical consequences of an emerging epidemic. Clin Microbiol Rev. 2010; 23(3):616-87. [DOI:10.1128/ CMR.00081-09] [PMID] [PMCID]

[9] Vinodhkumaradithyaa A, Uma A, Shirivasan M, Ananthalakshmi I, Nallasivam P, Thirumalaikolundusubramanian P. Nasal carriage of methicillinresistant Staphylococcus aureus among surgical unit staff. Jpn J Infect Dis. 2009; 62(3):228-9. [PMID]

[10] Ghaznavi-Rad E, Nor Shamsudin M, Sekawi Z, van Belkum A, Neela V. A simplified multiplex PCR assay for fast and easy discrimination of globally distributed staphylococcal cassette chromosome mec types in meticillin-resistant Staphylococcus aureus. J Med Microbiol. 2010; 59(10):1135-9. [DOI:10.1099/jmm.0.021956-0] [PMID]

[11] Alavi R, Darvishi M, Izadi M, Hami A, Hattami H. [Determination of the staphylococcus aureus nasal carriers prevalence and antibiotic resistance pattern in Surgical wards staff (Persian)]. Iran Infec Trop Med J. $2005 ; 2005: 43-6$.

[12] Askarian M, Zeinalzadeh A, Japoni A, Alborzi A, Memish ZA. Prevalence of nasal carriage of methicillin-resistant Staphylococcus aureus and its antibiotic susceptibility pattern in healthcare workers at Namazi Hospital, Shiraz, Iran. Int J Infect Dis. 2009; 13(5):241-7. [DOI:10.1016/j. ijid.2008.11.026] [PMID]

[13] Tafaroji J, Aghaali M, Heydari H. An investigation of the frequency of Staphylococcus aureus Nasal carriers and its antibiotic susceptibility pattern in the staff of different wards of Qom Hazrat Masumeh hospital, 2015, Iran. Qom Univ Med Sci J. 2017; 10(11):79-84. http://journal.muq. ac.ir/article-1-1337-en.html

[14] Nikbakht M, Hassan Nagad S, Rezazade B, Nagizadeh Baghi A, Gorbani $\mathrm{F}$, Faraji $\mathrm{F}$, et al. Antibiotic resistance pattern of isolated strains of staphy from personnel nasal specimens in Meshgin Shahar Valiasr hospital. $J$
Ardabil Univ Med Sci. 2009; 9(1):80-8. http://jarums.arums.ac.ir/article1-293-en.html

[15] Oguzkaya-Artan M, Baykan Z, Artan C, Avsarogullari L. Prevalence and risk factors for methicillin resistant Staphylococcus aureus carriage among emergency department workers and bacterial contamination on touch surfaces in Erciyes University Hospital, Kayseri, Turkey. Afr Health Sci. 2015; 15(4):1289-94. [DOI:10.4314/ahs.v15i4.31] [PMID] [PMCID]

[16] Saito G, Thom J, Wei Y, Gnanasuntharam P, Gnanasuntharam P, Kreiswirth $N$, et al. Methicillin-resistant Staphylococcus aureus colonization among health care workers in a downtown emergency department in Toronto, Ontario. Can J Infect Dis Med Microbiol, 2013; 24(3):57-60. [DOI:10.1155/2013/349891] [PMID] [PMCID]

[17] Al-Humaidan OS, El-Kersh TA, Al-Akeel RA. Risk factors of nasal carriage of Staphylococcus aureus and methicillin-resistant Staphylococcus aureus among health care staff in a teaching hospital in central Saudi Arabia. Saudi Med J. 2015; 36(9):1084-90. [DOl:10.15537/ smj.2015.9.12460] [PMID] [PMCID]

[18] Eveillard M, Martin Y, Hidri N, Boussougant Y, Joly-Guillou ML. Carriage of methicillin-resistant Staphylococcus aureus among hospital employees: prevalence, duration, and transmission to households. Infect Control Hosp Epidemiol. 2004; 25(2):114-20. [DOI:10.1086/502360] [PMID]

[19] Baldwin NS, Gilpin DF, Hughes CM, Kearney MP, Gardiner DA, Cardwell $\mathrm{Ch}$, et al. Prevalence of methicillin-resistant Staphylococcus aureus colonization in residents and staff in nursing homes in Northern Ireland. J Am Geriatr Soc. 2009; 57(4):620-6. [DOI:10.1111/ j.1532-5415.2009.02181.x] [PMID]

[20] Elie-Turenne MC, Fernandes $H$, Mediavilla JR, Mathema B, Singh A, Cohen TR, et al. Prevalence and characteristics of Staphylococ cus aureus colonization among healthcare professionals in an urban teaching hospital. Infect Control Hosp Epidemiol. 2010; 31(6):574-80. [DOI:10.1086/652525] [PMID]

[21] Julian T, Singh A, Rousseau J, Weese JS. Methicillin-resistant staphylococcal contamination of cellular phones of personnel in a veterinary teaching hospital. BMC Res Notes. 2012; 5(1):193. [DOI:10.1186/17560500-5-193] [PMID] [PMCID]

[22] Rasti F, Asghari E, Shahsavarinia K, Motazedi Z, Dehgani L. Microbia contamination of health care workers' mobile phones in Sina hospital, Tabriz. Hayat. 2016; 22(2):128-37. http://hayat.tums.ac.ir/article1-1434-en.html 